\title{
A case of pregnancy after liver transplantation and literature review
}

\begin{abstract}
The number of patients who are undergoing liver transplantation is increasing as well as their quality of life and chance for pregnancy. In numerous articles the effect of pregnancy on solid organ transplant recipients has previously been described. In this study a 35-year-old woman (gravida2, para 1) with a history of cryptogenic cirrhosis underwent liver transplantation. She had an uncomplicated postoperative course and was clinically stable on tacrolimus (level $7.9 \mathrm{ng} / \mathrm{mL}$ ) and prednisolone with no episodes of allograft rejection since transplant. Approximately 8 months after the operation, she became pregnant. Throughout the pregnancy, the transplant team as well as a perinatologist and obstetrician with a special interest in high-risk pregnancy closely followed her progress. Within this period no deterioration in graft functions or maternal and fetal complications were observed. She experienced an uncomplicated pregnancy. At 38 weeks of pregnancy, she delivered a healthy infant who had APGAR scores of seven and nine at five and ten minutes, respectively. Birth weight was $2.86 \mathrm{~kg}$, which was appropriate for gestational age. At eight month follow up the infant shows normal development, and there has been no deterioration in the grafts' function. This article shows that pregnancy appears to be safe following liver transplantation, but careful monitoring and pharmacotherapy are pivotal.
\end{abstract}

Keywords: liver transplantation, pregnancy, graft rejection, immuno suppression
Volume 5 Issue 4 - 2017

\author{
Soheila Milani,' Ebrahim Khaleghi² \\ 'Surgical Oncology Research Center, Mashhad University of \\ Medical Sciences, Iran \\ ${ }^{2}$ Organ Transplant Center, Mashhad University of Medical \\ Sciences, Iran
}

\begin{abstract}
Correspondence: Soheila Milani, Surgical Oncology Research Center, Imam Reza Hospital, Faculty of Medicine, Mashhad University of Medical Sciences, Mashhad, Iran, Tel +98 5I 3802 2677, Fax +985138519868,

Emaildrmilanis7@gmail.com,drmilani_s@yahoo.com
\end{abstract}

Received: June 25, 2017 | Published: July 14, 2017
Abbreviations: LT, liver transplantation; BMI, body mass index; ESLD, end stage liver disease; MELD, model for end stage liver disease; HPA, hypothalamic pituitary adrenal; ALT, alanine aminotransferase; AST, aspartate amino transferase

\section{Introduction}

It is estimated that women constitute more than $30 \%$ of liver transplantation (LT) recipients and about $8 \%$ are of reproductive age and $5 \%$ are pediatric females who will mostly survive into adulthood. It has been reported that $80 \%-90 \%$ of women at reproductive age after LT have a chance to become pregnant. ${ }^{2}$ However, Some $26 \%$ of solid-organ transplant recipients were advised against pursuing pregnancy by their physician in a recent survey. ${ }^{3}$ Unfortunately, some women and physicians remain misinformed about the feasibility and outcomes of pregnancy post transplantation. It is noteworthy that pregnancy following liver transplantation is feasible, although transplant patients have an increased risk of maternal and neonatal pregnancy-related complications, including prematurity and low birth weight, postpartum graft loss. Therefore, careful monitoring by a specialized multidisciplinary team is crucial. This article presents a case of a unplanned pregnancy after LT with overall good maternal and fetal outcomes. Our findings provide insights into the clinical implications of pregnancy after liver transplantation.

\section{Case presentation}

A thirty-five-year-old female with end-stage liver disease (ESLD) secondary to cryptogenic cirrhosis (BMI=30, MELD score $=18$ ) underwent deceased donor LT in May 2015. The deceased donor was a 24-year-old woman with a declaration of brain death after subarachnoid hemorrhage. The liver transplant operation was performed in 5hours, and the liver graft functioned well postoperatively. The patient received an immunosuppressive protocol consisting of tacrolimus, mycophenolate mofetil, and steroids, starting on postoperative day (POD) 1 . Recovery from transplant was uneventful. At about 6months after her LT, mycophenolate mofetil was discontinued and the immunosuppressive protocol continued with tacrolimus and prednisolone. After transplantation, her liver function tests were all normal, and she had no hypertension. Two months later she had an unplanned pregnancy. The next evaluation showed that she had no hypertension, no deterioration in the liver functions and foetal development, and amniotic fluid and Doppler evaluation of the umbilical artery were all within the normal range. Throughout the pregnancy, a specialized multidisciplinary team closely followed her progress. Tacrolimus doses were adjusted based on close monitoring of the levels throughout the pregnancy, whereas prednisolone doses remained stable. Tacrolimus levels were stable throughout pregnancy and within the accepted target range of 6-10. For the patient in addition to all mandatory standard tests during pregnancy also careful monitoring of weight gain plus abdomen circumference between checkups were performed. During the pregnancy, all clinical and laboratory tests, especially levels of bilirubin, alanine aminotransferase (ALT), and aspartate aminotransferase (AST) were acceptable.

At 38 weeks of gestation, the overall evaluation of the foetus and mother showed no contraindication for vaginal delivery, so the patient was managed for vaginal delivery after administering vaginal dinoprostone. She had an uncomplicated vaginal delivery and gave birth to a healthy infant, who had APGAR scores of seven and nine 
at five and ten minutes, respectively. Birth weight was $2.86 \mathrm{~kg}$, which was appropriate for gestational age. At eight month follow up the infant shows normal development, and there has been no deterioration in the grafts' function.

\section{Discussion}

Amenorrhea is common among females with ESLD, with reported rates of up to $50 \% .{ }^{4}$ Amenorrhea in liver disease is multifactorial, often dependent on the etiology of the underlying liver disease and frequently related to malnutrition, which is particularly common in cirrhotic patients. Reports propose that menstrual function often promptly returns in $85 \%$ of women within 6months of LT. ${ }^{5}$ Thus, preconception counselling is an essential part of pregnancy planning in the liver transplant recipient of childbearing age. There is limited evidence regarding the safest and most effective method of contraception following LT for prevention of an unplanned pregnancy. Obviously, this issue should be considered in future research. A large populationbased study also reported on obstetrical outcomes in liver transplant recipients. ${ }^{6}$ There have been no reported associations between liver transplantation and increased rates of maternal mortality.

Pregnancy induced hypertension has been common among liver transplant recipients, with rates varying from $2 \%$ to $43 \%$. Preeclampsia is also commonly seen, with rates between $2 \%$ and $22 \%$. The reported rates of gestational diabetes mellitus (GDM) have varied widely, from $0 \%$ to $37.5 \%$. Graft rejection rates during pregnancy varied from $0 \%$ to $20 \%$. Factors associated with graft loss within five years were reported in the National Transplantation Pregnancy Registry in 2009. Important predictors included Caucasian race, viral hepatitis as etiology of liver failure, age $<18$ years at time of transplant and rejection during pregnancy. ${ }^{7}$ Anyway, in LT recipient the consequence of rejection during the peripartum period are significant and increase the risk of graft loss, immuno suppression must be maintained throughout pregnancy with careful monitoring of drug concentrations.

Most studies have reported that the rates of cesarean delivery among liver transplant recipients were consistently higher than the general non transplant population (20\% to $100 \%)$ and uncomplicated vaginal delivery rates (without assistance) ranged widely $(0 \%$ to $50 \%) .{ }^{6}$ The causes of this wide range should be investigated in future studies. As well, fetal deaths are more common in liver transplant recipients. Stillbirths were quite uncommon, with rates of $0 \%$ to $2 \%$ being reported. Low birth weight $(<2500 \mathrm{~g})$ is also a common complication, with rates ranging from $4.8 \%$ to $57 \%$. Rates of fetal distress are also increased (10.3\% to $40 \%)$ during these pregnancies. Overall, congenital abnormalities are uncommon and minimally increased, with rates of $0 \%$ to $16.7 \% .^{6}$ Thus comparing these reports with our results suggests that the outcome of pregnancy after liver transplantation should be revised. Finally, after LT pregnancy should be advised in women who are maintained on low-dose immunosuppressive therapy with proper allograft function, which is defined as stable levels of bilirubin, alanine aminotransferase (ALT), and aspartate aminotransferase (AST). As the patient with poor or unstable liver function are particularly susceptible to rejection if they become pregnant. ${ }^{8}$

In conclusion, pregnancy in liver transplant recipients is associated with a higher risk of adverse maternal and fetal outcomes. However, increasing experience with careful management of these patients has enabled to minimize these risks.

\section{Acknowledgements}

None.

\section{Conflict of interest}

The author declares no conflict of interest.

\section{References}

1. Ramirez CB, Doria C. Pregnancy after liver transplantation. Best Pract Res Clin Obstet Gynaecol. 2014;28(8):1137-1145.

2. Jabiry-Zieniewicz Z, Dabrowski FA, Pietrzak B, et al. Pregnancy in the liver transplant recipient. Liver Transpl. 2016;22(10):1408-1417.

3. Humphreys RA, Wong HH, Milner R, et al. Pregnancy outcomes among solid organ transplant recipients in British Columbia. J Obstet Gynaecol Can. 2012;34(5):416-424.

4. Esposti SD. Pregnancy in patients with advanced chronic liver disease. Clin Liv Dis. 2014;4(3):62-68.

5. Parolin MB, Rabinovitch I, Urbanetz AA, et al. Impact of successful liver transplantation on reproductive function and sexuality in women with advanced liver disease. Transplant Proc. 2004;36(4):943-944.

6. Coffin CS, Shaheen AA, Burak KW, et al. Pregnancy outcomes among liver transplant recipients in the United States: a nationwide case-control analysis. Liver Transpl. 2010;16(1):56-63.

7. Coscia LA, Constantinescu S, Moritz MJ, et al. Report from the National Transplantation Pregnancy Registry (NTPR): outcomes of pregnancy after transplantation. Clin Transpl. 2010. p. 65-85.

8. Coffin CS, Shaheen AA, Burak KW, et al. Pregnancy outcomes among liver transplant recipients in the United States: a nationwide case-control analysis. Liver Transpl. 2010;16(1):56-63. 